This is a peer-reviewed, accepted author manuscript of the following article: Mauro, F., Benci, A., Ferrari, V., \& Della Valentina, E. (2021). Dynamic positioning analysis and comfort assessment for the early design stage of large yachts. International Shipbuilding Progress. https:// doi.org/10.3233/ISP-210508

\title{
Dynamic Positioning analysis and comfort assessment for the early design stage of large yachts
}

\author{
Francesco Mauro a,b,*, Aron Benci ${ }^{\mathrm{b}}$ Victor Ferrari $^{\mathrm{c}}$ and \\ Enrico Della Valentina ${ }^{c}$ \\ ${ }^{a}$ Maritime Safety and Research Centre MSRC, University of Strathclyde, United Kingdom \\ E-mail: francesco.mauro@strath.ac.uk \\ ${ }^{\mathrm{b}}$ Department of Engineering and Architecture, University of Trieste, Italy \\ E-mail: aronbenci@gmail.com \\ ' Ship Department, Maritime Research Institute of the Netherlands MARIN,the Netherlands \\ E-mails:vfferrari@marin.nl,e.valentina@marin.nl
}

In some specific environmentally protected areas, conventional mooring systems cannot be used by large yachts for stationing at anchor and therefore, the adoption of a dynamic positioning system is required. It becomes then necessary to evaluate the station keeping capabilities of a yacht since the earlydesign stage. Adopting a quasi-static approach, it is possible to perform a standard capability analysis, as commonly done for the offshore industry, obtaining a capability plot as output. However, capability plots are referring to specific wind-wave correlation that are not covering all the possible wave combinations present in a sea area. Here, it is proposed to use a scatter diagram approach for the dynamic positioning analysis of a large yacht, considering the specific sea areas where the yacht shall operate, in order to figure out the downtime period of the DP system per each sea area. The proposed method can be coupled with traditional ship motions analysis, leading to a combination between comfort assessment and DP predictions. In the present work, use has been made of a traditional displacement yacht $72 \mathrm{~m}$ long, comparing five different DP system configurations and evaluating an enhanced comfort ranking combining ISO AWI-22834 guidelines for large yachts with ISO AWI-22822 DP analysis.

Keywords: Large yachts, Comfort on-board, DP predictions, Early-design stage, Ship motions

\section{Introduction}

In the yacht industry, it is possible to subdivide pleasure craft into small crafts from 8 to $24 \mathrm{~m}$ and large yachts above $24 \mathrm{~m}$, as referred by ISO/TC 8/SC 12 . One of the most important targets in large yachts' design is the achievement of an adequate comfort level while cruising or stationing at anchor [1]. These aspects are related to a wide range of possible sources of discomfort, including also the effect of ship motions and accelerations in different sea states. In this sense, there is a lack of standards and criteria specific for the motions-related comfort on-board assessment of

\footnotetext{
*Corresponding author. E-mail: francesco.mauro@strath.ac.uk.
} 
large yachts. Criteria are available in the literature for various kind of ships, as for example the Nordforsk ones [2], or specific studies on large cruise ships [3]. However, no one of them is specific for yachts or directly associated to leisure activities at anchor. To fill this gap, AWI-22834 working group led by MARIN has been established within ISO to produce a comparative scale of comfort among yachts that can be used for technical and commercial benefit [4] during the early design stage.

However, besides traditional seakeeping challenges, during stationing additional issues can be given by the mooring system. In case the yacht is expected to operate in environmentally protected areas, it can be required to provide the stationing of the vessel without traditional mooring lines, thus adopting a dynamic positioning (DP) system [5]. In such a case, several aspects that are not usually taken into account in large yacht design should be considered, such as a proper determination of the environmental loads acting on the vessel. Moreover, the necessity to use a DP system, allows the designer to investigate multiple possible solutions for the propulsive system and for the thruster devices to install on-board. To efficiently compare possible propulsive solutions since the early-design stage, it is necessary to provide a sufficiently reliable and fast calculation method to assess DP capability and rank the different design solutions. To this end, use can be made of quasi-static calculations, providing the necessary balance between reliability and calculation time for an initial design stage [6]. The traditional output of a quasi-static simulation is the so-called DP capability plot [7], reporting a maximum sustainable wind speed per each ship encounter angle. However, a capability plot is representative of standard environmental conditions which are based on offshore industry necessities.

For a large yacht, the working environment and the reference design loads are not comparable with offshore industry ones. Therefore, it is advisable to adopt a different approach for the DP capability prediction of such type of vessels. An appropriate set of environmental conditions for yacht DP analysis is still under discussion [8], and different possibilities may be chosen. Being yachts sailing and stationing at mostly in the Caribbean or Mediterranean Sea [9], it is possible to identify the characteristic working environment by means of the specific wave statistics of these areas. Then, the DP performances can be evaluated on a scatter diagram base [10], providing the downtime period of the DP system per each selected sea area. Adopting this approach, it is possible to rank the operability of the DP system according to an index of merit [4], comparing the DP capabilities of different yachts or different design solutions. With this approach it is possible to have both DP and seakeeping calculations for the same environmental conditions.

In this study, the MARIN yacht [11] has been chosen as reference hull, having at disposal wind loads measurements and seakeeping experiments. The original yacht propulsive configuration (propeller/rudder) has been analysed, considering different management of the rudders control. The results have been compared with other possible design solutions, including the adoption of a POD based propulsion system. The different DP system solutions are compared both in terms of standard capability plots and newly proposed downtime approach, providing, in this last case, a DP merit 
index per each configuration. Besides, the MARIN yacht comfort has been analysed according to AWI-22834 guidelines for the stationing conditions. The DP analysis has been combined together with comfort criteria to verify whether the station keeping abilities may influence the comfort rating of the vessel. Two possible integration ways have been introduced and compared in terms of resulting comfort rating.

\section{Comfort on-board of large yachts}

In the design of a pleasure craft, the concept of comfort is of paramount importance, however a unique and complete definition of comfort does not exist as it includes subjective feelings of individuals. Moreover, the perception of comfort can be influenced by many factors, which are also dependent upon time to exposure, intensity of the phenomenon but also upon gender, age, previous experience and mental state of the subject. The most relevant factors for a yacht can be summarized as follows:

- noise and vibrations;

- motions and postural stability;

- smell;

- on-board amenities, food and drink;

- other factors (weather, temperature, humidity, etc.).

This study is focused on comfort related to ship motions, thus on the application of specific criteria associated to ship motions and acceleration. It is then necessary to identify the most relevant criteria associated to ship motions that can be referred to the judgement of comfort or discomfort on-board of a large yacht.

General criteria used for commercial vessels are available (an overview is given in Table 1), even though they were originally intended for certain operations on-board of either navy-vessels or passenger ships. The operations and the life on-board of a yacht are more similar to passenger ships ones rather than navy activity, therefore it is more reasonable to adopt criteria close to the one used for passenger vessels. However, specific criteria for passenger ships [3] adopted inside MARIN requires the calculation of the Motion Illness Rating (MIR), which adopts coefficients not available in the literature. Furthermore, its determination requires many parameters that could not be available in an early design stage.

To overcome this issue and to provide criteria of easy applicability for a large yacht, the AWI-22834 guidelines have been developed, to propose a framework for the determination of comfort levels for large yachts.

\subsection{The AWI-22834 guidelines}

To overcome the lack of precise standards and criteria specifically devoted for large yachts, ISO working group AWI 22834 led by MARIN has been established. 
The main goal of this working group is to produce a comparative scale of comfort among yachts that can be used for technical and commercial benefit. The process goes through the following steps:

- identification of yacht specific criteria for comfort;

- environmental condition selection;

- operative condition selection (speed, encounter angle);

- identification of on board location of interest;

- evaluation of criteria satisfaction for all conditions.

As previously mentioned, available criteria for ship motion related comfort (Table 1) refer to other kind of vessels, therefore a selection of the most suitable criteria has been carried out. In the specific, Effective Gravity Angle (EGA) and Motion Sickness Index (MSI) have been chosen for the comfort assessment, adopting the following formulations:

$$
E G A_{L}=\arctan \left(\frac{\ddot{\eta}_{L}}{\ddot{\zeta}_{L}+g}\right)
$$

where low-case $P$ denotes the calculation point, $g$ is the gravity accelerations and $\ddot{\zeta}_{L}$ and $\ddot{\eta}_{L}$ are the absolute vertical and lateral acceleration in the calculation point. Equation (1) can be interpreted both in time domain and frequency domain; in the latter case, RMS values are considered.

For MSI, the following formulation has been taken into account [12]:

$$
\begin{aligned}
M S I_{L} & =100 \Phi\left(Z_{\zeta}\right) \Phi\left(Z_{t}^{\prime}\right) \\
Z_{\zeta} & =2.128 \log \ddot{\zeta}_{P} / g-9.277 \log \hat{\omega}_{\zeta}-5.809 \log \hat{\omega}_{\zeta}^{2}-1.851 \\
Z_{t}^{\prime} & =1.134 Z_{\zeta}+1.989 \log T_{e}-2.904 \\
\Phi(Z) & =\frac{1}{\sqrt{2 \pi}} \int_{-\infty}^{Z} e^{\frac{-x^{2}}{2}} \mathrm{~d} x
\end{aligned}
$$

where $T_{e}$ is the exposure time in minutes and $\hat{\omega}_{\zeta}$ is the frequency in Hertz of the absolute vertical acceleration $\ddot{\zeta}$ spectrum' peak. This is a simplified version of MSI, taking into account only the vertical acceleration.

This choice has been made because of the necessity to have a right balance between the complexity of the criteria formulation and the need to have a sufficiently board indicator to describe comfort. According to the proposers, the combination between MSI and EGA makes it possible to have a single indicator covering both the need of people to look for support (but also for tipping or sliding of objects) and the percentage of people on-board that will suffer from seasickness after a certain exposure time. 
The AWI working group proposes also the identification of the environmental conditions, the locations on-board and the fulfilment criteria for EGA and MSI. The selection of environmental conditions is based on the assumption that most of large yachts operates mostly in the Western Mediterranean and Caribbean Seas [9], using a combined wave scatter diagram of the two sea areas according to annual statistics. The analysis is limited to the wave heights between 1 and 2 meters: the waves with the higher occurrence in the combined scatter diagram, and ensuring a fair compromise with at anchor performances of vessels relatively short (less than about $60 \mathrm{~m}$ ) and longer ones [22]. An overview of the resulting environmental conditions is given in Figure 1. All the irregular wave conditions refer to long crested sea approximation and are modelled with a JONSWAP wave spectrum.

The analysis should be carried out on 5 reference locations among the yacht, representative of 5 areas of interest for operations and leisure on-board:

1. Owners Cabin (OC);

2. Dining Area (DA);

3. Wheel House (WH);

4. Crew Area (CA);

5. Beach Club (BC).

Each of the above mentioned area should be identified by considering the geometrical centre of that space on-board, adding 1.2 meters to the reference deck height for the $z$ coordinate. The analysis should be carried out for bow quartering seas over starboard, analysing also the mirrored position along longitudinal axis for points not located on the yacht diametral plane. Analysis should be carried out for two speeds: 0 and 12 knots. For all the mentioned conditions, the fulfilment criteria have been set to $E G A_{P}<2 \mathrm{deg}$ and $M S I<10 \%$ with $T_{e}$ equal to 1 hour.

Within this framework, it is then possible to identify the downtime period of the comfort criteria fulfilment at each speed $V$ as follows:

$$
\begin{aligned}
& O P_{\text {comf }_{V}}=\sum_{i=1}^{N_{T_{z}}} \sum_{j=1}^{N_{L}} p_{T_{z i}} p_{L_{j}} I_{\text {conf }} i j \\
& I_{\text {comf }}= \begin{cases}1 & \text { if } M S I_{L}<10 \% \wedge E G A_{L}<2 \mathrm{deg} \\
0 & \text { otherwise }\end{cases}
\end{aligned}
$$

where $p_{T_{z}}$ is the marginal distribution of the wave periods as shown in Figure 1 and $p_{L}$ is a weight function given at each location of interest. The preliminary AWI proposal is considering an equal weight within all the 5 locations (i.e. $N_{L}=5$ ). As final outcome, based on the values of $O P_{c o m f_{V}}$ a rating is given on the base of a 5 stars scale. This relation is given in Table 2 . The obtained rating can be global or local: in the latter case, it is sufficient to neglect the loop on locations from equation (6). 


\section{Dynamic positioning on large yachts}

Dynamic positioning is a typical issue of offshore industry, where nowadays almost all vessel are requested to operate stationing in deep water environment with or without the aid of mooring lines. However this offshore technology can be applied also to other vessels, once they are required to autonomously keep a predetermined position, thus also on large yachts.

To determine the DP capabilities of a vessel, two different approaches can be followed: a dynamic approach or a quasi-static one. Dynamic simulations, even though they can be implemented also in simplified forms, are more oriented to the reproduction of the effective system mounted on-board [13], thus more indicated for an advanced design stage [14]. For an early-design stage, the quasi-static approach is preferable, giving the opportunity to analyse and compare multiple design solutions with a relatively short calculation time $[15,16]$. These considerations made for offshore vessels can be shifted also to the yacht industry, therefore in this study a quasistatic approach has been selected for the analyses.

The quasi-static DP problem consists in the resolution of the equilibrium of forces and moments acting on the yacht on the horizontal plain. Considering the reference system of Figure 2, centred in the vessel midpoint $\mathrm{O}$, the equilibrium is:

$$
\begin{cases}\sum_{i=1}^{N_{P}} F_{X_{P_{i}}} & =F_{X_{E N V}}+F_{X_{E X T}} \\ \sum_{i=1}^{N_{P}} F_{Y_{P_{i}}} & =F_{Y_{E N V}}+F_{Y_{E X T}} \\ \sum_{i=1}^{N_{P}}\left(F_{Y_{P_{i}}} x_{P_{i}}-F_{X_{P_{i}}} y_{P_{i}}\right) & =M_{Z_{E N V}}+M_{Z_{E X T}}\end{cases}
$$

where $F_{X_{P}}$ and $F_{Y_{P}}$ are the longitudinal and lateral forces provided by each of the $N_{P}$ actuators installed on-board. $x_{P}$ and $y_{P}$ are the coordinates of the actuators (i.e. propulsors or tunnel thrusters) with respect to vessel midpoint $\mathrm{O}$. In the right term of system (8), external and environmental forces appear. The external loads may be used to simulate any additional force or moment acting on a vessel, as for the case of a large yacht additional mooring lines, or can be neglected from the equilibrium. Here, aiming to study a stand-alone DP system, only environmental loads have been considered and are analysed in detail for the MARIN yacht test case.

\subsection{Thrust allocation}

The resolution of the quasi-static DP problem requires solving system (8) considering the actuator forces and directions as the problem unknowns. The resolution of system (8) is not an easy task to perform. Generally, the unknowns of the problem are $2 N_{S T}+N_{T T}$ where $N_{S T}$ is the number of steerable actuators (including the case of propeller plus rudder) and $N_{T T}$ is the number of fixed tunnel thrusters.

Thus, having system (8) only 3 equations, the problem admits infinite possible feasible solutions. Therefore, it is necessary to adopt a suitable allocation procedure 
to distribute the thrust with the correct orientation among the units to satisfy the equilibrium [17]. Here, a non-linear optimisation method has been used [18], considering the following non-linear objective function:

$$
\begin{aligned}
\min (z) & =\sum_{i=1}^{N_{P}} T_{i}^{\frac{2}{3}}=\sum_{i=1}^{N_{P}}\left(\sqrt{{F_{X_{P i}}}^{2}+{F_{Y_{P i}}}^{2}}\right)^{\frac{2}{3}}= \\
& =\sum_{i=1}^{N_{P}}\left(\sqrt{\left(T_{i} \cos \alpha_{i}\right)^{2}+\left(T_{i} \sin \alpha_{i}\right)^{2}}\right)^{\frac{2}{3}}
\end{aligned}
$$

This formulation is representative of the sum of absorbed power of each actuator, and can be written as a function of thrust components $F_{X_{P}}, F_{Y_{P}}$ or as a function of thrust $T$ and thrust orientation $\alpha$. The non-linear optimization technique allows to manage also non-linear constraints, therefore, once the $T, \alpha$ unknowns setting is selected, system (8) becomes a set of non-linear constraints that suits with the adopted optimisation algorithm. In this study, use has been made of the $T, \alpha$ unknowns, because it suits better with the modelling adopted for the propeller/rudder, where the developed forces in $x$ and $y$ directions are described as follows:

$$
\begin{aligned}
& F_{X_{R}}=T\left(1-C_{X} \alpha^{2}\right) \\
& F_{Y_{R}}=T C_{Y} \alpha
\end{aligned}
$$

where $C_{X}$ and $C_{Y}$ are given by DNV regulations [19]. In such a way, the rudder angle becomes one of the unknowns and the differences between dependent and independent rudder control can be easily implemented in the resolution algorithm.

\subsection{Capability plots approach}

The standard way to represent DP calculations is by means of the so-called capability plots [7]. These diagrams are representative of the maximum sustainable wind speed $V_{W}$ the DP system can face at each encounter angle $\chi$ using the on-board thrust devices. The capability plots are obtained by considering a specific wind-wave correlation curve, which is associating at each $V_{W}$ a precise value of $H_{s}$ and $T_{z}$. Current speed $V_{C}$ is kept constant and collinear with wind and wave loads. To have a more reliable prediction, environmental loads are multiplied by a dynamic allowance coefficient (usually ranging between 1.20 and 1.25) to take into account dynamic effects in the quasi-static procedure. An example of capability plot is given in Figure 3, highlighting the maximum $V_{W}$ at each $\chi$ ranging between $0^{\circ}$ to $360^{\circ}$ degrees for a sample vessel, using the IMCA wind-wave correlation.

Capability plots offer an easy way to compare capabilities of different DP configurations on the same vessel or compare DP systems mounted on-board of different 
units. However, they refer to specific environmental conditions dictated by the windwave correlation adopted for the assessment. Therefore, this method is not suitable to match the same calculation conditions typical of comfort assessment analysis.

\subsection{Scatter diagram approach}

A different strategy to perform quasi-static calculation is the adoption of a scatter diagram approach [10]. This method allows a designer to have a more complete vision of the DP system performances in the sea environment of interest for the yacht. As mentioned, capability plots refer to a specific wind-wave correlation, thus they did not ensure that DP calculations cover all the possible combination of $H_{s}$ and $T_{z}$ that may occur in a sea area. By performing dedicated prediction in each cell of a wave scatter diagram, it is then sure that all the possible sea states have been analysed.

With this novel approach, the wind speed in each cell of the scatter diagram can be derived from the Pierson-Moskowitz wave spectrum, relating $V_{W}$ to $H_{s}$ and $T_{z}$. Therefore, each cell of the scatter diagram will have a different calculation wind speed. For the current loads, the same settings of the capability plots have been chosen considering a current speed of $0.75 \mathrm{~m} / \mathrm{s}$ collinear with wind and waves. Being also scatter diagram approach a quasi-static method, a dynamic allowance coefficient can be applied to environmental loads.

This method allows to evaluate the downtime period for the DP system in a selected sea area by means of a DP operability index having the following form:

$$
\begin{aligned}
& O P_{D P}=\sum_{k=1}^{N_{\chi}} p_{\chi_{k}} \sum_{i=1}^{N_{H_{S}}} \sum_{j=1}^{N_{T_{z}}} p_{w_{i j}} I_{D P_{i j k}} \\
& I_{D P}= \begin{cases}1 & \text { if position is kept } \\
0 & \text { otherwise }\end{cases}
\end{aligned}
$$

where $p_{\chi}$ is the occurrence of each encounter angle the vessel is asked to keep and $p_{w}$ is the joint probability of $H_{s}$ and $T_{z}$ given by the selected scatter diagram. In Figure 4 an example of the scatter diagram approach is reported for one heading condition, highlighting the differences with conditions used for the capability plots determination.

It can be observed that, considering only one heading and one $H_{s}$ value, equation (12) has the same form of equation (6) adopted by AWI-22834 for comfort analysis. Therefore, the scatter diagram approach is capable to evaluate DP performance in the same environmental conditions of comfort analysis for large yachts. 


\section{The reference yacht}

The vessel selected for this investigation is the MARIN yacht [11], a reference large yacht hull where the results of seakeeping tests and wind loads measurements are at disposal for research purposes. For such a reason, this yacht has been selected as basis platform for the performed explorative study on comfort analysis and DP system capabilities on large yachts. An overview of the main characteristics of the yacht is reported in Table 3. Hereafter, the preliminary ship motion calculations and the DP configurations considered for this study are given together with the environmental loads determination on the reference yacht as a starting point for the DP and comfort analysis.

\subsection{Ship motion calculations}

To evaluate the comfort assessment on a large yacht according to AWI-22834 guidelines (section 2.1), it is necessary to evaluate the ship motions of the vessel under analysis. There are several ways to predict ship motions, having different levels of accuracy and calculation/realisation time. The most accurate way possible to evaluate ship motion is still given by model tests, but it is almost impossible performing them in an early design stage. However, model test data on reference ship can be used to validate computational tools (like viscous CFD, diffraction or strip theory codes) that better suits for preliminary calculations.

This approach has been followed for the MARIN yacht, where experimental measurements are at disposal for ship motions. Calculations have been performed by means of Maxsurf 3D diffraction panel code, comparing motion RAOs with available experiments for beam and bow quartering seas, without the use of active stabilisers. Results are reported in Figure 5 for heave and roll motion, showing a good agreement between numerical and experimental curves, thus giving sufficient reliability to the ship motion analysis.

\subsection{DP configurations}

In this explorative study on large yachts, different DP configurations have been considered to study the system performance changes on the same base hull, in this case the MARIN yacht. The selected configurations are representative of standard propulsive layouts already present on-board of traditional large yachts, plus the addition of alternative solutions oriented to increase vessel low speed maneuverability, and therefore, probably more suitable to improve the station keeping ability of a yacht. Hereafter a brief description of the proposed configurations is given. It must be observed, that it is not possible to adopt a configuration fully derived from offshore applications (i.e. application of large azimuth thruster) to not compromise internal layout and yacht propulsive operative profile. An overview of the configurations is given in Figure 6, together with size and positioning of the thrust devices given according to reference system defined in Figure 2. The main details of the rudders used in conventional propulsive configurations are reported in Table 4. 
4.2.1. C1: conventional propulsion with dependent rudders and bow tunnel thruster

This configuration (referred hereafter as $\mathrm{C} 1$ in the text) is a standard propulsive configuration for a twin screw large yacht. It is composed by a conventional propellers/rudders system and a tunnel thruster at the bow. The rudder control system allows to orientate the rudders only with the same angle. Configuration $\mathrm{C} 1$ is the simplest kind of DP system that can be implemented on-board, by simply using the same machinery installed for maneuvering and propulsion

\subsubsection{C2: conventional propulsion with dependent rudders and bow/stern tunnel thrusters}

This configuration (referred hereafter as C2 in the text) is a variation of configuration $\mathrm{C} 1$. The propulsion remains a conventional one for twin-screw yachts, considering dependent rudder management strategy. The main difference with respect to configuration $\mathrm{C} 1$ is related to the tunnel thrusters. In this specific case, instead of one bow tunnel thruster as in C1, 2 tunnels have been considered, one on the bow and one on the stern. The stern tunnel thruster can be integrated into the skeg, or, in case more space is needed can be of retractile type. In order to have the same amount of power installed on-board, the thruster sizes are reduced compared to previous configurations.

\subsubsection{C3: conventional propulsion with independent rudders and bow tunnel thruster}

This configuration (referred hereafter as C3 in the text) is a variation of previously described configuration $\mathrm{C} 1$. The yacht is still equipped with a conventional propulsion system. However, the rudder control is somewhat different, allowing to set different angles between the two rudders. The bow thruster mounted on-board is the same of configuration $\mathrm{C} 1$.

\subsubsection{C4: Conventional propulsion with independent rudders and bow/stern tunnel thrusters}

This configuration (referred hereafter as $\mathrm{C} 4$ in the text) is a variation of configuration $\mathrm{C} 3$. The propulsion remains a conventional one for twin-screw yachts, considering an independent rudder management strategy. As for configuration $\mathrm{C} 2$, an additional stern tunnel thruster has been considered, changing the size of the fixed thrusters.

\subsubsection{C5: Podded propulsion with bow tunnel thruster}

This last configuration (referred hereafter as C5 in the text) is representative of an unconventional propulsive solution for a large yacht. Instead of a conventional twin screw propulsion with conventional rudders, two steerable PODs are mounted on the vessel stern. Even though limitations are given for the maximum steering angle of a POD in sailing conditions, for zero speed application they can be oriented among all directions, avoiding mutual interactions with the adoption of forbidden zones in thrust allocation algorithm. The propulsive loads are the same of previous configuration, therefore, the pod maximum thrust has been considered comparable 
with the standard propellers. At the bow, one tunnel thruster is present with the same characteristics of the one installed for configurations $\mathrm{C} 1$ and $\mathrm{C} 2$.

\subsection{Environmental loads}

The determination of environmental loads for the vessel under analysis is one of the most important steps for the DP predictions. In this study, environmental forces are given by the collinear and simultaneous action of wind, waves and current. The determination of such loads is not an easy task in an early design stage, especially for large yachts, due to the lack of specific simplified methods, that are available for offshore vessel forms only. Hereafter, the procedure adopted to determine the environmental loads is given, comparing also the force coefficients used for the MARIN yacht with simplified method derived from the offshore industry.

The wind loads are determined at each encounter angle $\chi$ on the bases of nondimensional coefficients according to the following formulations:

$$
\begin{aligned}
F_{X_{W}} & =\frac{1}{2} \rho_{a i r} A_{F} V_{W}^{2} C_{X_{W}}(\chi) \\
F_{Y_{W}} & =\frac{1}{2} \rho_{a i r} A_{L} V_{W}^{2} C_{Y_{W}}(\chi) \\
M_{Z_{W}} & =\frac{1}{2} \rho_{a i r} A_{L} L_{P P} V_{W}^{2} C_{M_{W}}(\chi)
\end{aligned}
$$

where $A_{F}$ and $A_{L}$ are the frontal and lateral area of the yacht superstructures, $L_{P P}$ is the length between perpendiculars, $V_{W}$ the wind speed in $\mathrm{m} / \mathrm{s}$ and $\rho_{\text {air }}$ the air density. The determination of wind loads coefficients for a large yachts in the early design stage, is not an easy task due to the lack of informations on the real yacht superstructures at disposal. It is possible to adopt model experiments on reference superstructures (like the Blendermann database [20]) or statistical methods based on wind data collections [21]. Another alternative could be the adoption of simplified methods derived from the offshore industry and applied by Classification Societies as reference for preliminary DP calculations [19]. For the MARIN yacht, wind tunnel measurements are available for the $C_{X_{W}}, C_{Y_{W}}$ and $C_{M_{W}}$ coefficients. In Figure 7 a comparison is given among the above mentioned methods, highlighting how measurements differs from simplified approaches for the $C_{X_{W}}$ and $C_{M_{W}}$, while $C_{Y_{W}}$ matches reasonably well.

The current loads are also determined starting from non-dimensional coefficients, using formulations analogue to (14), (15) and (16) to obtain dimensional loads. However, for current purposes, superstructure areas are substituted by wetted surface $S$, reference is made to current speed $V_{C}$ and water density for salt water $\rho_{w}$. For current loads, really simple formulations are available in the literature $[19,22]$ referring to offshore applications. For the MARIN yacht geometry, current loads coefficients 
have been determined by means of double body RANS calculations. In Figure 8 the coefficients obtained with CFD are compared with the DNV procedure for DP calculations. As previously observed for the wind, the $C_{Y_{C}}$ coefficient is the only one having the same trend with both approaches, even though for current there is a difference above $30 \%$ on the maximum amplitude value.

For wave loads, irregular long crested sea conditions have been considered, starting from drift coefficients, derived from diffraction calculations as reported in section 4.1, and modelling the irregular waves with a spectrum:

$$
\begin{aligned}
& F_{X_{w}}=\rho_{w} g \nabla^{\frac{1}{3}} \int_{0}^{\infty} C_{X_{w}}(\chi, \omega) S_{\zeta}(\omega) \mathrm{d} \omega \\
& F_{Y_{w}}=\rho_{w} g \nabla^{\frac{1}{3}} \int_{0}^{\infty} C_{Y_{w}}(\chi, \omega) S_{\zeta}(\omega) \mathrm{d} \omega \\
& M_{Z_{w}}=\rho_{w} g \nabla^{\frac{2}{3}} \int_{0}^{\infty} C_{M_{w}}(\chi, \omega) S_{\zeta}(\omega) \mathrm{d} \omega
\end{aligned}
$$

where $\nabla$ is the vessel volume of displacement, $g$ the acceleration of gravity and $S_{\zeta}$ is the wave amplitude spectrum expressed as a function of circular wave frequency $\omega$. There are not simplified methods to directly determine frequency dependent drift force coefficients. Therefore, in an early-design stage, an alternative could be the application of simple methods to directly derive the forces $[19,22]$. The general method given by DNV is suitable for short crested sea, which means the wave spectrum $S_{\zeta}$ is no more dependent only by $\omega$ but also by $\chi$. In Figure 9 an example is given of the loads obtained applying DNV methods against the ones coming from diffraction coefficients for long or short crested irregular waves. The comparison is made with a JONSWAP spectrum for $H_{s}$ of 1.5 metres and $T_{z}$ of 6 seconds (the most probable according to AWI-22834 marginal distribution for $T_{z}$ ), using for short crested sea the parameters implemented in the DNV method. The wave forces generated with the DNV method are more conservative than direct calculation coming from diffraction theory also for the long crested sea case.

Despite the absence of simplified methods specific for large yachts, wind and current environmental loads can be estimated in an early design stage also by means of methods derived from the offshore industry. Wave loads are overestimated compared to direct calculations and this may lead to underestimation of DP capability performances. These considerations based on the MARIN yacht test case are not enough to derive a general guidelines, however highlight an issue that should be properly analysed with further investigations.

For such a case it has been decided to consider the loads coming from experiments and direct calculations for the DP analyses. 


\section{DP and comfort analysis for the MARIN yacht}

Having defined the methods identified for the DP and comfort assessment in the early design stage of a large yacht, it is then possible to apply it on the MARIN yacht. The DP and comfort analysis will be firstly individually analysed; in a second phase, the two analyses are combined to obtain a single index of merit.

\subsection{Comfort assessment}

The comfort assessment for the MARIN yacht has been performed according to the AWI-22834 guidelines for the zero speed condition. The 5 locations for the comfort assessment are reported in Figure 10. Without having the effective internal layout, the selection of such coordinates for the calculation points has been decided based on MARIN internal statistics on the most recurrent calculation areas on yacht with comparable size.

In Figure 11 it is possible to observe the $M S I$ and $E G A$ variations in the five locations among the $T_{z}$ considered by the guidelines. It can be observed that, for the MARIN yacht, the only limiting comfort criteria is related to the MSI in the crew area CA, for $T_{z}$ around 6 seconds. The EGA criterion is always satisfied. This results in a top comfort rating according to AWI-22834 rating-system.

To have a comparison with other criteria related to comfort, the analysis has been carried out on the same environmental conditions, applying Nordforsk criteria for cruise liners (see Table 1). Here limits are given on vertical and lateral accelerations together with the RMS of roll motion. The study has been performed on the same locations used for AWI analysis. Figure 12 reports the criteria evaluation for the five locations. In this case the limitation is given by the vertical acceleration (which is related to $M S I$ ), however the limiting value of the Nordforsk criterion is more restrictive than the one given by AWI-22834 guidelines. In Table 5 the final rating obtained by the application of the two guidelines are given, highlighting the extremely restrictive nature of Nordforsk criteria for vertical acceleration. For the MARIN yacht, only the dining area DA satisfies the criteria, suggesting that the limiting vertical acceleration is probably too restrictive for large yacht.

\subsection{DP calculations}

The traditional analysis approach based on capability plots has been carried out as initial comparison among different DP system options. This standard capability analysis has been performed for the five DP system configurations and the obtained results are shown in Figure 13. For the calculations, IMCA wind-wave correlation has been used, a current speed of $0.75 \mathrm{~m} / \mathrm{s}$ collinear with wind and waves and a dynamic allowance coefficient of 1.25 . It can be noted that, globally, the higher capability is given by configuration $\mathrm{C} 5$. Configuration $\mathrm{C} 1$ is giving the lower capability envelope, being not extremely effective for beam and stern-quartering seas. It can be 
observed that by introducing a stern thruster (config. C2) the capability of the vessel improves significantly throughout the whole encounter angles, having performance comparable with independent rudder (config. C3). The additional stern thruster is not really effective for the independent rudder configuration (config. C4), in fact, for stern-quartering seas, the capability envelope is quite lower than configuration C3, while for head seas they remain comparable.

The capability plot is for sure a valuable method to compare different DP design solutions. However, from the capability plot analysis it is not possible to clearly rank the five design solutions, because the effectiveness of the system is changing at each heading angle and only the maximum sustainable wind speed $V_{W}$ can be addressed, without having a clear understanding of the DP system behaviour in the real working environment of the yacht.

For such a reason, the scatter diagram approach has been also applied to the five design solutions for the MARIN yacht. For the current loads, the same settings of the capability plots have been chosen considering a current speed of $0.75 \mathrm{~m} / \mathrm{s}$ collinear with wind and waves. Being also scatter diagram approach a quasi-static method, a dynamic allowance coefficient of 1.25 has been also applied for these calculations. The irregular waves have been simulated with a JONSWAP spectrum, varying $\gamma$ elongation parameter according to $H_{s}$ and $T_{z}$ combination. Moreover, it should be considered that a large yacht is not requested to keep a predefined heading while holding the position, therefore, it is more interesting to focus the analyses on the head seas, rather than on the whole $360 \mathrm{deg}$ as in the capability plots. Therefore, scatter diagram calculations have been performed from the range of $0^{\circ}$ to $60^{\circ}$ degrees in steps of $15^{\circ}$. As already mentioned, the scatter diagram approach allows to identify the cells of a scatter diagram where the DP system is capable to hold the vessel in position, thus is dividing the sea area in two zones, one where the vessel can and another where cannot hold the position (according to $I_{D P}$ defined in equation (13)).

The analysis can be applied to specific sea areas considering multiple headings (thus evaluating the $O P_{D P}$ index according to equation (12)) or considering custom wave conditions for only one heading as in the AWI-22834 comfort assessment. In this study both approaches have been followed. In Figure 14 an example is given for Western Mediterranean sea; the figure shows the data relative to headings of $15^{\circ}, 30^{\circ}$ and $45^{\circ}$ degrees. Head seas, $0^{\circ}$ degrees, has been omitted because the DP system is always successful for all the five configurations. $60^{\circ}$ degrees has been omitted because is not giving additional visual information compared to $45^{\circ}$ degrees case. From the plotted data, it immediately appears that configuration C5 has the highest station keeping ability. The same comments given from the capability plot analyses can be also extended to the scatter diagram approach. Due to the intrinsic nature of this calculation approach, in case the same type of wave spectrum is used, the cells (i.e. the combination of $H_{s}$ and $T_{z}$ ) where the DP system holds position remains the same among all possible sea areas. Therefore, by changing scatter diagram, only the joint occurrence $p_{w}$ is changing, giving a different $O P_{D P}$ value. 
In Table 6 the value of the $O P_{D P}$ index is reported for the different 5 configurations, considering the operation in Area 26 and Area 47 for all the calculated headings. Besides, also the operability index for the AWI-22834 condition is reported. All the data are given in terms of $O P_{D P}$ index, with its relative correspondence with AWI-22834 star-rating system (Table 2).

The global data in Table 6 reports a higher $O P_{D P}$ value for Area 26 compared to Area 47. The 5 tested DP system configurations are suffering for high wave heights and for high periods, therefore the operability of the system will reflect the joint wave distribution of each area. Area 47 is characterised by lower wave heights but higher periods compared to Area 26, having a higher wave density for wave-parameters combinations where DP system is not capable to hold position.

\subsection{Combined analysis}

The reported comfort and DP analyses are dissociated between each other. However, adopting a scatter diagram approach, it is possible to have DP and seakeeping calculations performed with the same environmental conditions. Moreover, the downtime-based rating system proposed by AWI-22834 guidelines well suits also for DP calculations. It is then possible to integrate the two analyses, leading to a global index of merit for a large yacht.

In this study, two possible integration methods are proposed, both based on comfort and DP analyses performed as described in the previous sections.

\subsection{DP as an additional criterion}

A first way to integrate DP into the comfort analysis framework proposed by AWI22834 is to consider the DP assessment as an additional criteria for the determination of $O P_{\text {comf }}$ parameter (equation (6)). In such a case, the parameter $I_{\text {comf }}$ becomes:

$$
I_{\text {comf }}= \begin{cases}1 & \text { if } M S I_{L}<10 \% \wedge E G A_{L}<2 \operatorname{deg} \wedge I_{D P}=1 \\ 0 & \text { otherwise }\end{cases}
$$

where $I_{D P}$ is calculated for the AWI-22834 environmental conditions. This formulation is filtering out possible sea states where the comfort performances are satisfied but the vessel is not able to keep position.

Applying this method to the MARIN yacht, it results in eliminating from the acceptable $H_{s}$ and $T_{z}$ combinations sets found with the comfort analysis all the higher $T_{z}$ cells, resulting in a lower $O P_{\text {comf }}$ value. The obtained data are reported in Table 7. With this new notation, only configuration C5 maintain a comfort rating of five stars. All the other configuration have a reduced rating, especially $\mathrm{C} 1$, having a DP totally inefficient for AWI-22834 heading. 
This way to include DP in the comfort analysis may be too much penalising for simple DP systems, giving too much impact to DP performances instead of intrinsic motions related comfort of the yacht. Therefore a second option has been analysed in the following section.

\subsection{DP for optimal comfort heading}

A second option for the integration of DP results into an early-design comfort analysis is to consider the DP system for is effective duty, which means to keep a desired heading leading to a more comfortable life on-board.

The previous proposal was forcing the DP system to keep the heading $\chi$ of $45^{\circ}$ degrees, condition not $100 \%$ favourable for all the DP system configurations here presented. This results in a general down-rate of the global ranking of the vessel, especially for simple DP systems. However, being a large yacht not required to keep the predetermined position, it can be stated that a yacht with DP system mounted on-board can operate at a minimum motion heading, so increasing the comfort of the vessel. This can be achieved by introducing an additional calculation heading to the comfort and DP analyses.

By adopting the AWI-22834 guidelines for comfort rating, the conditions where $M S I$ and $E G A$ have less impact corresponds to head sea. Therefore the final $O P_{\text {comf }}$ can be evaluated as follows:

$$
O P_{\text {comf }}=\frac{\left.O P_{\text {comf }}\right|_{\chi=45^{\circ}}+\left.O P_{\text {comf }}\right|_{\chi=0^{\circ}}}{2}
$$

In such a case, the values of $O P_{\text {comf }}$ for $45^{\circ}$ and $0^{\circ}$ degrees should be inclusive of the DP analysis, thus with an $I_{\text {comf }}$ evaluated according to equation 20 . The results obtained applying this alternative inclusion method for DP are reported in Table 8. The final rating for the 5 configurations is still lowered compared to the pure motion ones, however there is a more fair comparison among different DP systems. Configurations $\mathrm{C} 1$ and $\mathrm{C} 3$ are the ones who benefit the most from this method with an increase of the rating by two additional stars. For the other configurations the improvement is more contained although still relevant.

\section{Configuration ranking}

As final consideration, the five tested DP configurations of the MARIN yacht can be ranked, based on the above described indices of merit. Being the motions related comfort evaluated with the AWI-22834 guidelines high for this large yacht, all the differences among the configuration are only related to DP system. For the analysed conditions, a final ranking can be summarised as follows: 
(1) Configuration C5: this configuration is without any doubt the most indicate to maximize the DP capability of a large yacht. The adoption of POD units increases the possibility to face the incoming loads by orienting the thrust with more freedom respect to a propeller/rudder system.

(2) Configurations $C 4$ and C2: these two configurations are the second-best option to have good station keeping capability on a large yacht. The installation of a stern thruster in combination with the independent or dependent rudder management is offering a good performance for the station keeping of the yacht. The system hold capacity is poor only for headings $>45^{\circ}$ degrees, especially for high $T_{z}$.

(3) Configuration C3: this configuration, with independent rudders and a single bow tunnel thruster, has lower station keeping ability respect to the previous one, but it can still be considered sufficient. The hold capacity is poor only at large heading angles.

(4) Configuration C1: the standard propulsive configuration of a yacht has poor station keeping ability for both the tested sea areas. The system grants sufficient performances only for heading angle less than $30^{\circ}$ degrees in all the tested scenarios.

This final ranking is subject to all the assumptions reported in the previous sections and to the adoption of the environmental loads specific for the MARIN yacht. However, this ranking is respecting the general trends observed for offshore industry, therefore the adoption of other environmental loads or the application on different yachts may lead to the same qualitative results, changing only the indexes magnitude.

\section{Conclusions}

In the present study, an explorative analysis has been performed on the MARIN yacht to evaluate the impact on station keeping capability of different possible DP system design solutions and its integration with AWI-22834 guidelines for comfort assessment. The tested configurations include standard propulsion layouts for a large yacht and more advanced ones, including different management of the rudder control, the addition of stern thrusters or the adoption of a podded propulsion.

For the comfort analysis, the AWI-22834 guidelines for large yachts have been followed, comparing the differences of the proposed limiting criteria with other available in literature on the same environmental conditions. The analysis highlights that the AWI criteria are less restrictive than others, but probably more suitable to establish a comparison between large yachts.

The DP analysis has been firstly performed with standard capability plots, stating that this method is not really suitable to analyse the system performances considering the effective sea environment the yacht will face during service. On the contrary a scatter diagram approach allows to analyse and compare multiple configurations 
ranking it according to predefined merit index in different sea areas. The AWI ranking method based on stars has been used to obtain a homogeneous result with comfort analysis on yachts. This allows to integrate also the DP results with the comfort analysis. Two different methods have been presented and compared, showing that a fair integration can be achieved by considering more vessel headings in the combined analysis and using DP as additional limiting criterion for comfort.

The obtained results are specific for the tested case of the MARIN yacht, however the results of this explorative study may be a starting point for more general considerations. The ranking of the proposed configurations shows that the best configuration is the one with pods (C5), granting the maximum combined index of comfort. The traditional configuration (C1) with dependent rudders and bow tunnel thruster is the worse one. An attractive solution is given by the installation of a stern thruster as per the configuration $\mathrm{C} 2$ and $\mathrm{C} 4$. In fact, the adoption of a stern thruster is giving a larger improvement with respect to the selection between dependent or independent rudders.

This preliminary study highlights some aspects that needs to be further investigated for the establishment of a general procedure for DP and comfort assessment of large yacht in the early design stage. In the specific, a detailed study on the determination of general environmental loads is needed, together with a reliable criteria for acceptability for yacht DP performances. Besides, additional features can be incorporated, performing dedicated studies on power consumption, the energy management on board, the impact of vibration noise induced by different propulsion systems and underwater radiated noise.

\section{References}

[1] C. Backer and K.M. Sweeney, Setting a standard for luxury and comfort, in: Proceedings of the Design, Construction and Operation of Super and Mega Yachts Conference, Genova, 2009.

[2] NORDFORSK, Assessment of Ship Performance in a Seaway, Technical Report, NORDFORSK, 1987.

[3] R. Grin, J. van Heerd and V. Ferrari, Hydrodynamic aspects in the design of passenger vessels, in: Proccedings of RINA, Royal Institution of Naval Architects - Design and Operation of Passenger Ships, 2013.

[4] E. Della Valentina, ISO/AWI 22834 Large yachts - quality assessment of life on-board, Technical Report, MARIN, 2019

[5] R. Calver and J. Roy, Dynamic Positioning capabilities for superyachts, 2015.

[6] O. Smogeli, N. Trong, B. Borhaug and L. Pivano, The next level DP capability analysis, in: Proceedings of Dynamic Positioning Conference, 2013.

[7] IMCA, Specification for DP capability plots, Technical Report, IMCA, 2000.

[8] H. Cozijn, ISO/AWI 22822 Large yachts - quality assessment and acceptance criteria - Dynamic positioning systems on large yachts, Technical Report, MARIN, 2019.

[9] P.T. van Loon and P. van Zoon, From operational profile to hybrid propulsion, in: Proceedings of the 24th HISWA Symposium on Yacht Design and Constructions, Amsterdam, 2016

[10] F. Mauro and J. Prpić-Oršić, Determination of a DP operability index for an Offshore vessel in early-design stage, Ocean Engineering 195 (2020), 106764. 
[11] V. Ferrari, T. Gornicz, A. Kisjes and F. Quadvlieg, Influence of skeg on ship manoeuvrability at high and low speeds, in: 14th International Symposium on Practical Design of Ships and other Floating Structures PRADS 2019, Japan, 2019.

[12] NATO, STANAG 4154 Common Procedures for Seakeeping in the Ship Design Process, Technical Report, NATO, 2000.

[13] J.J. Serraris, Time-domain analysis for DP simulations, in: Proceedings of OMAE Conference, Honolulu, 2009.

[14] A. Aalberts, R. Kuipers, F. van Walree and R. Jansen, Developments in dynamic positioning systems for offshore stationkeeping and offloading, in: Proceedings of ISOPE Conference, The Hague, 1995.

[15] J. Wichers and R. van Dijk, Benefits of using assisted DP for deepwater mooring systems, in: Proceedings of Offshore Technology Conference, 1999.

[16] F. Mauro and R. Nabergoj, Optimal thruster location on offshore DP vessels, International Shipbuilding Progress 66(2) (2019), 145-162.

[17] E. van Daalen, H. Cozijn, C. Loussouran and P. Hemker, A generic optimization algorithm for the allocation of DP actuators, in: Proceedings of the International Conference on Offshore Mechanics and Artic Engineering OMAE, 2011.

[18] F. Mauro and R. Nabergoj, Advantages and disadvantages of thruster allocation procedures in preliminary dynamic positioning predictions, Ocean Engineering 123 (2016), 96-102.

[19] DNV-GL, DNVGL-ST-0111: Assessment of station keeping capability of dynamic positioning vessels, Technical Report, DNV-GL, 2018.

[20] W. Blendermann, Wind loading of ships - collected data from wind tunnel tests in uniform flow, Technical Report, Institut für Shiffbau der Universität Hamburg, 1996.

[21] W. Bledermann, Estimation of wind loads on ships in wind with a strong gradient, in: 14th International Conference on Offshore Mechanics and Artic Engineering OMAE, New York, 1995.

[22] API, API 2P-RP 1984, Technical Report, American Petroleum Institute, 1984. 
Fig. 1. Western Mediterranean (top left) and Caribbean Sea (top right) scatter diagram and combined marginal wave distribution (bottom) considered by AWI 22834.

Fig. 2. Reference system for DP and comfort analyses.

Fig. 3. Example of DP capability plot for a sample vessel.

Fig. 4. DP scatter diagram approach example (left) for a heading of $45^{\circ}$ degrees and comparison with associated points on a capability plot (right) based on wind-wave correlations.

Fig. 5. Heave and Roll motion RAOs for the MARIN yacht: experimental and numerical values.

Fig. 6. Analysed DP system configurations for MARIN yacht.

Fig. 7. Wind loads coefficients for the MARIN yacht: experimental data and empiric-statistics methods.

Fig. 8. Current loads coefficients for the MARIN yacht: CFD calculations and empiric-statistics method.

Fig. 9. Wave loads for the MARIN yacht: diffraction calculations and empiricstatistics method.

Fig. 10. Locations for comfort analysis on the MARIN yacht.

Fig. 11. MSI and EGA evaluation for MARIN yacht according to AWI-22834 guidelines.

Fig. 12. Application of Nordforsk comfort criteria to the MARIN yacht for the AWI-22834 environmental conditions.

Fig. 13. Capability plots comparison between DP system configurations for MARIN yacht.

Fig. 14. Scatter diagram comparison between DP system configurations for MARIN yacht in area 26. 
Table 1

Comfort related ship motion criteria applicable for passenger ships.

\begin{tabular}{ccc}
\hline Criterion name & Quantity & Criteria fulfilment \\
\hline Effective Gravity Angle & $E G A$ & $R M S<2 \mathrm{deg}$ \\
ISO2631-1 & $M S I$ & $M S I<10 \%$ \\
Comfort rating for passenger ships & $M I R$ or $C R$ & $M I R<10$ (for passenger) \\
& Vertical acceleration & $M I R<20$ (for crew) \\
Nordforsk & Lateral acceleration & $R M S<0.02 g$ \\
& roll & $R M S<2 \mathrm{deg}$ \\
Vibration Dose Value & $V D V$ & $V D V<1$ \\
\hline
\end{tabular}

Table 2

AWI 22834 star-rating system.

\begin{tabular}{cc}
\hline OP $_{\text {comf }}(\%)$ & stars \\
\hline $0-20$ & $\star \star \star \star \star$ \\
$20-40$ & $\star \star \star \star \star$ \\
$40-60$ & $\star \star \star \star \star$ \\
$60-80$ & $\star \star \star \star \star$ \\
$80-100$ & $\star \star \star \star \star$ \\
\hline
\end{tabular}

Table 3

MARIN yacht main characteristics.

\begin{tabular}{lll|llcr}
\multicolumn{1}{c}{ Quantity } & & Value & \multicolumn{1}{c}{ Quantity } & & Value & 34 \\
\cline { 1 - 5 } Length between perpendiculars & $L_{P P}$ & $72.00 \mathrm{~m}$ & Displacement mass (salt water) & $\Delta$ & $1730.1 \mathrm{t}$ & 35 \\
Length on waterline & $L_{W L}$ & $73.35 \mathrm{~m}$ & Wetted surface (bare hull) & $S$ & $1094.7 \mathrm{~m}^{2}$ & 36 \\
Length overall submerged & $L_{O S}$ & $79.06 \mathrm{~m}$ & Frontal area (superstructures) & $A_{F}$ & $147.1 \mathrm{~m}^{2}$ & 37 \\
Breadth & $B$ & $12.40 \mathrm{~m}$ & Lateral area & $A_{L}$ & $854.1 \mathrm{~m}^{2}$ & 38 \\
Mean draught & $T_{M}$ & $3.312 \mathrm{~m}$ & Lateral area centre (from O) & $x_{W}$ & $3.49 \mathrm{~m}$ & 39 \\
Long. centre of gravity (from O) & $x_{G}$ & $-1.88 \mathrm{~m}$ & Inertia radius around $x$ axis & $K_{x x}$ & $4.64 \mathrm{~m}$ & 41 \\
Lat. centre of gravity (from O) & $y_{G}$ & $0.00 \mathrm{~m}$ & Inertia radius around $y$ axis & $K_{y y}$ & $20.00 \mathrm{~m}$ & 42 \\
Vertical centre of gravity (from O) & $z_{G}$ & $2.30 \mathrm{~m}$ & Inertia radius around $z$ axis & $K_{z z}$ & $20.52 \mathrm{~m}$ & 43 \\
\hline
\end{tabular}


Table 4

MARIN yacht rudders main characteristics.

\begin{tabular}{llc}
\hline \multicolumn{1}{c}{ Quantity } & & Value \\
\hline Number of rudders & & 2 \\
Average height & $b_{R}$ & $2.32 \mathrm{~m}$ \\
Average chord length & $c_{R}$ & $1.53 \mathrm{~m}$ \\
Geometric aspect ratio & $\lambda_{R}$ & 1.52 \\
Projected area & $A_{R}$ & $3.55 \mathrm{~m}^{2}$ \\
Longitudinal position (from O) & $x_{R}$ & $0.00 \mathrm{~m}$ \\
Lateral offset (from O) & $\left|y_{R}\right|$ & $3.00 \mathrm{~m}$ \\
Maximum rudder angle & $\alpha_{M A X}$ & $45.00 \mathrm{deg}$ \\
\hline
\end{tabular}

Table 5

MARIN yacht comfort rating according to AWI-22384 and Nordforsk criteria.

\begin{tabular}{ccccc}
\hline \multirow{2}{*}{ Location } & \multicolumn{2}{c}{ AWI-22834 } & \multicolumn{2}{c}{ Nordforsk } \\
& $O P_{\text {comf }}$ & rating & OP comf & rating \\
\hline OC & 100.00 & $\star \star \star \star \star$ & 1.96 & $\star \star \star \star \star$ \\
DA & 100.00 & $\star \star \star \star \star$ & 100.00 & $\star \star \star \star \star$ \\
WH & 100.00 & $\star \star \star \star \star$ & 1.96 & $\star \star \star \star \star$ \\
CA & 82.75 & $\star \star \star \star \star$ & 0.00 & $\star \star \star \star \star$ \\
BC & 100.00 & $\star \star \star \star \star$ & 1.96 & $\star \star \star \star \star$ \\
\hline GLOBAL & 96.55 & $\star \star \star \star \star$ & 21.18 & $\star \star \star \star \star$ \\
\hline
\end{tabular}
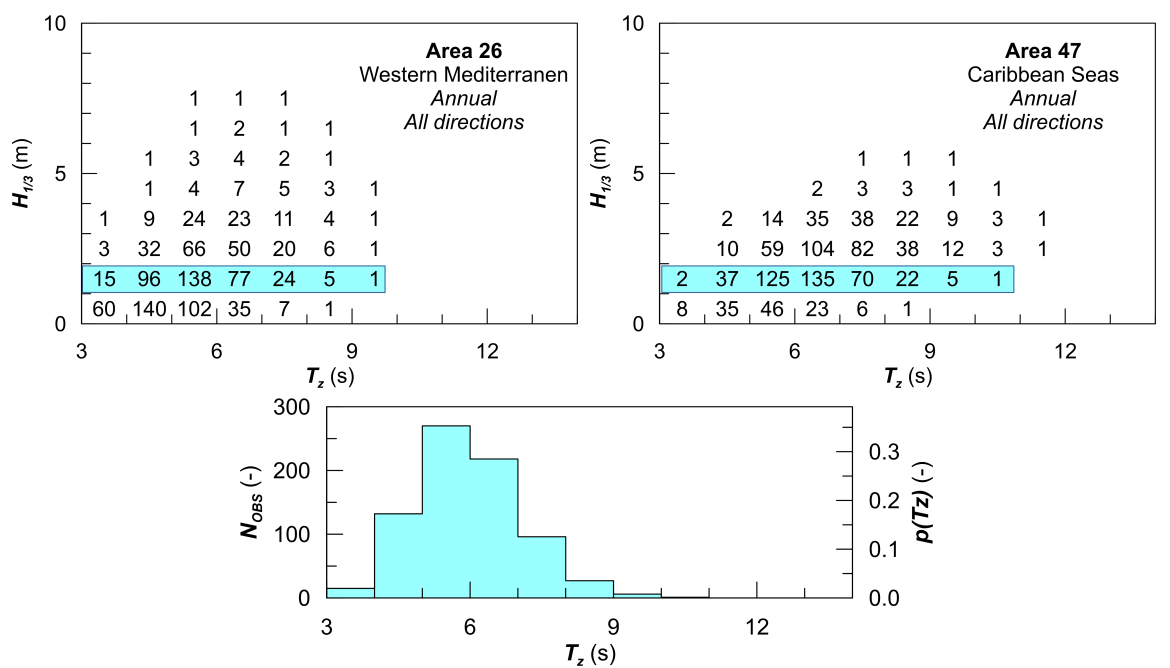

Fig. 1. Western Mediterranean (top left) and Caribbean Sea (top right) scatter diagram and combined marginal wave distribution (bottom) considered by AWI 22834 . 
Table 6

MARIN yacht configurations DP ratings.

\begin{tabular}{|c|c|c|c|c|c|c|c|c|c|c|}
\hline & \multicolumn{10}{|c|}{ Area 26 Western Mediterranean } \\
\hline & \multicolumn{2}{|c|}{$\chi=0^{\circ} \operatorname{deg}$} & \multicolumn{2}{|c|}{$\chi=15^{\circ} \operatorname{deg}$} & \multicolumn{2}{|c|}{$\chi=30^{\circ} \operatorname{deg}$} & \multicolumn{2}{|c|}{$\chi=45^{\circ} \operatorname{deg}$} & \multicolumn{2}{|c|}{$\chi=60^{\circ} \operatorname{deg}$} \\
\hline & $O P_{D P}$ & rating & $O P_{D P}$ & rating & $O P_{D P}$ & rating & $O P_{D P}$ & rating & $O P_{D P}$ & rating \\
\hline $\mathrm{C} 1$ & 100.00 & $\star \star \star \star \star$ & 99.62 & $\star \star \star \star \star$ & 23.34 & 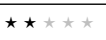 & 0.00 & 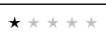 & 0.00 & 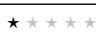 \\
\hline $\mathrm{C} 2$ & 100.00 & $\star \star \star \star \star$ & 99.82 & $\star \star \star \star \star$ & 92.09 & $\star \star \star \star \star$ & 58.09 & 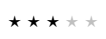 & 23.34 & 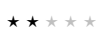 \\
\hline $\mathrm{C} 3$ & 100.00 & $\star \star \star \star \star$ & 99.65 & $\star \star \star \star \star$ & 88.46 & $\star \star \star \star \star$ & 48.59 & $\star \star \star \star \star$ & 19.81 & 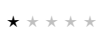 \\
\hline $\mathrm{C} 4$ & 100.00 & $\star \star \star \star \star$ & 99.82 & $\star \star \star \star \star$ & 92.48 & $\star \star \star \star \star$ & 62.68 & $\star \star \star \star \star$ & 31.28 & 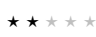 \\
\hline C5 & 100.00 & $\star \star \star \star \star$ & 99.97 & $\star \star \star \star \star$ & 97.10 & $\star \star \star \star \star$ & 82.36 & $\star \star \star \star \star$ & 66.13 & $\star \star \star \star \star$ \\
\hline \multicolumn{11}{|c|}{ Area 47 Caribbean sea } \\
\hline & \multicolumn{2}{|c|}{$\chi=0^{\circ} \operatorname{deg}$} & \multicolumn{2}{|c|}{$\chi=15^{\circ} \mathrm{deg}$} & \multicolumn{2}{|c|}{$\chi=30^{\circ} \mathrm{deg}$} & \multicolumn{2}{|c|}{$\chi=45^{\circ} \mathrm{deg}$} & \multicolumn{2}{|c|}{$\chi=60^{\circ} \operatorname{deg}$} \\
\hline & $O P_{D P}$ & rating & $O P_{D P}$ & rating & $O P_{D P}$ & rating & $O P_{D P}$ & rating & $O P_{D P}$ & rating \\
\hline $\mathrm{C} 1$ & 100.00 & $\star \star \star \star \star$ & 100.00 & $\star \star \star \star \star$ & 5.54 & 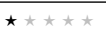 & 0.00 & 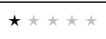 & 0.00 & 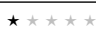 \\
\hline $\mathrm{C} 2$ & 100.00 & $\star \star \star \star \star$ & 100.00 & $\star \star \star \star \star$ & 84.77 & $\star \star \star \star \star$ & 28.43 & 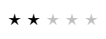 & 5.54 & 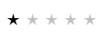 \\
\hline $\mathrm{C} 3$ & 100.00 & $\star \star \star \star \star$ & 100.00 & $\star \star \star \star \star$ & 73.70 & $\star \star \star \star \star$ & 19.80 & 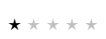 & 4.63 & 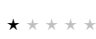 \\
\hline $\mathrm{C} 4$ & 100.00 & $\star \star \star \star \star$ & 100.00 & $\star \star \star \star \star$ & 85.76 & $\star \star \star \star \star$ & 33.06 & 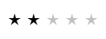 & 8.94 & 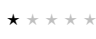 \\
\hline $\mathrm{C} 5$ & 100.00 & $\star \star \star \star \star$ & 100.00 & $\star \star \star \star \star$ & 96.54 & $\star \star \star \star \star$ & 61.95 & $\star \star \star \star \star$ & 38.10 & 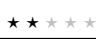 \\
\hline \multicolumn{11}{|c|}{ AWI-22834 conditions } \\
\hline & \multicolumn{2}{|c|}{$\chi=0^{\circ} \mathrm{deg}$} & \multicolumn{2}{|c|}{$\chi=15^{\circ} \operatorname{deg}$} & \multicolumn{2}{|c|}{$\chi=30^{\circ} \mathrm{deg}$} & \multicolumn{2}{|c|}{$\chi=45^{\circ} \mathrm{deg}$} & \multicolumn{2}{|c|}{$\chi=60^{\circ} \operatorname{deg}$} \\
\hline & $O P_{D P}$ & rating & $O P_{D P}$ & rating & $O P_{D P}$ & rating & $O P_{D P}$ & rating & $O P_{D P}$ & rating \\
\hline $\mathrm{C} 1$ & & & & & & & 0.00 & 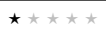 & & \\
\hline $\mathrm{C} 2$ & & & & & & & 54.84 & $\star \star \star \star \star$ & & \\
\hline $\mathrm{C} 3$ & & & & & & & 37.38 & 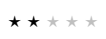 & & \\
\hline $\mathrm{C} 4$ & & & & & & & 61.88 & $\star \star \star \star \star$ & & \\
\hline $\mathrm{C} 5$ & & & & & & & 89.24 & $\star \star \star \star \star$ & & \\
\hline
\end{tabular}

Table 7

MARIN yacht configurations global ratings considering DP as additional limiting criterion.

\begin{tabular}{ccc}
\hline configuration & $O P_{\text {comf }}(\%)$ & rating \\
\hline C1 & 0.00 & $\star \star \star \star \star$ \\
C2 & 51.06 & $\star \star \star \star \star$ \\
C3 & 33.41 & $\star \star \star \star \star$ \\
C4 & 58.18 & $\star \star \star \star \star$ \\
C5 & 85.83 & $\star \star \star \star \star$ \\
\hline
\end{tabular}

Table 8

MARIN yacht configurations global ratings considering two headings.

\begin{tabular}{ccc}
\hline configuration & $O P_{\text {comf }}(\%)$ & rating \\
\hline C1 & 45.98 & $\star \star \star \star \star$ \\
C2 & 71.51 & $\star \star \star \star \star$ \\
C3 & 62.69 & $\star \star \star \star \star$ \\
C4 & 75.07 & $\star \star \star \star \star$ \\
C5 & 88.90 & $\star \star \star \star \star$ \\
\hline
\end{tabular}




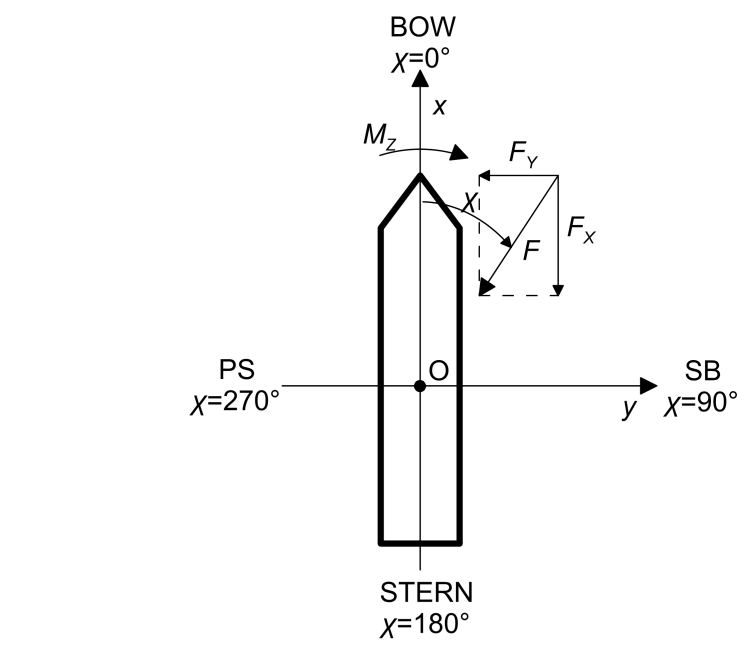

Fig. 2. Reference system for DP and comfort analyses.

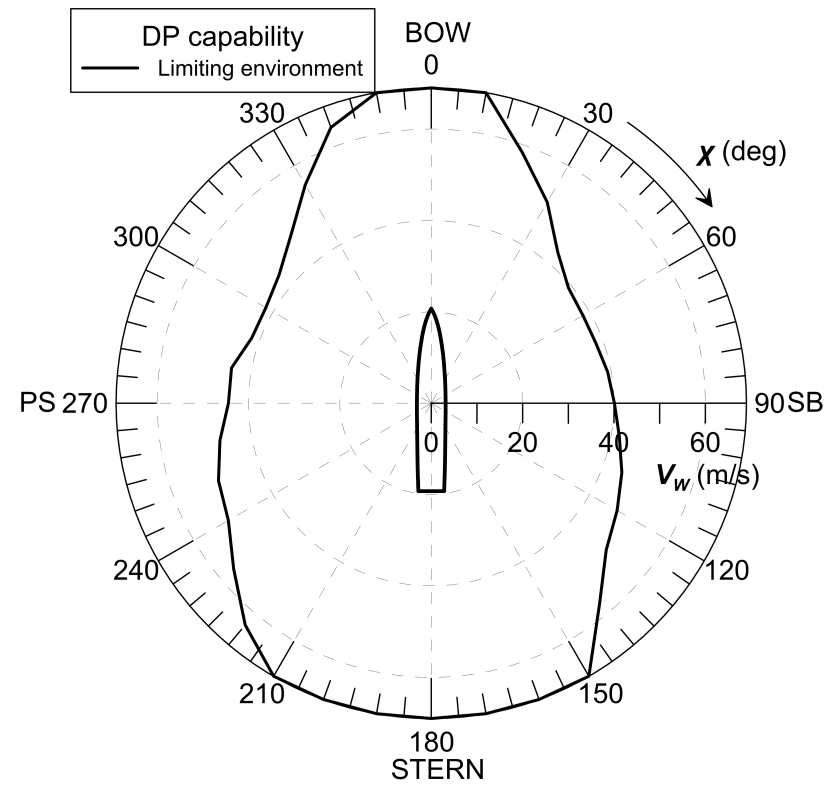

Fig. 3. Example of DP capability plot for a sample vessel. 

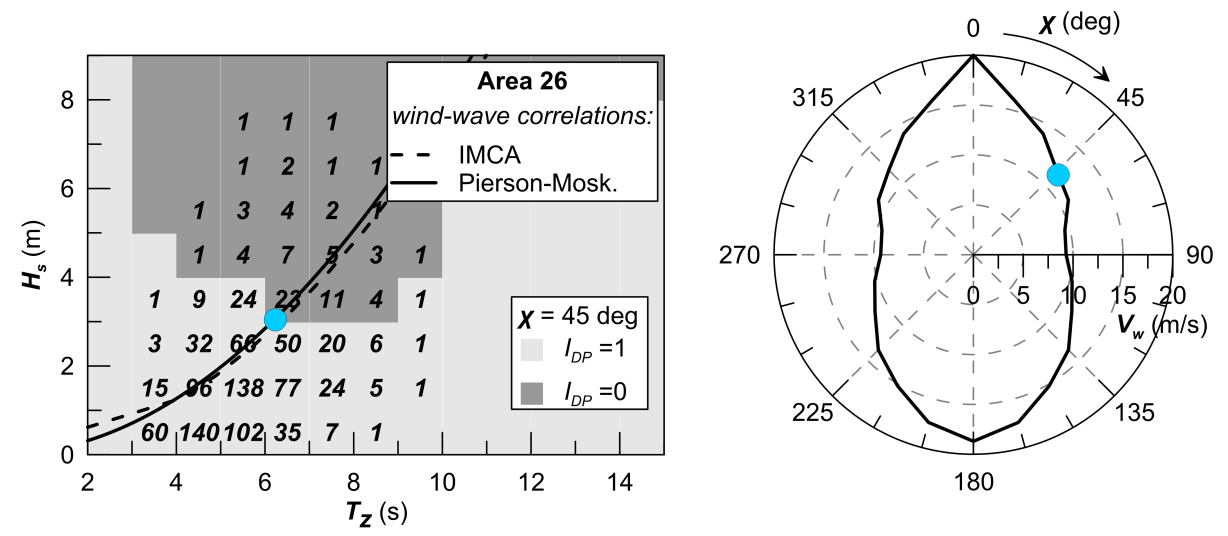

Fig. 4. DP scatter diagram approach example (left) for a heading of $45^{\circ}$ degrees and comparison with associated points on a capability plot (right) based on wind-wave correlations.
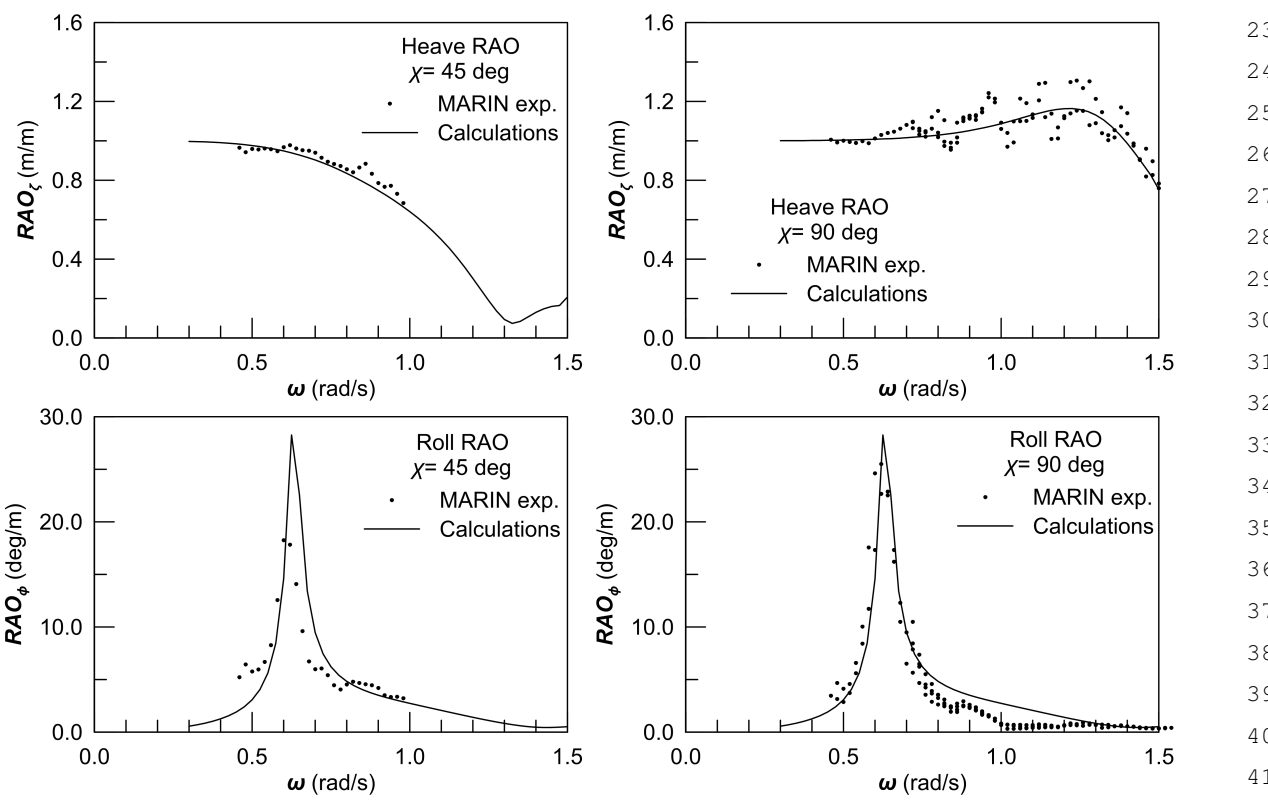

Fig. 5. Heave and Roll motion RAOs for the MARIN yacht: experimental and numerical values. 

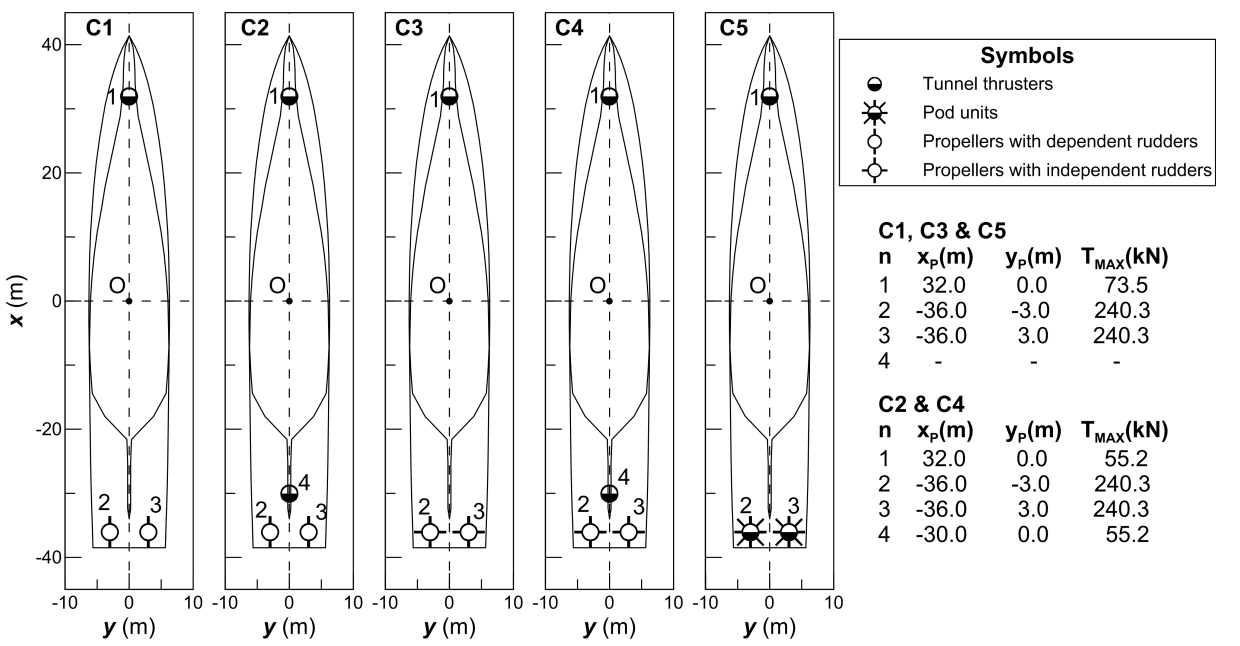

Fig. 6. Analysed DP system configurations for MARIN yacht.
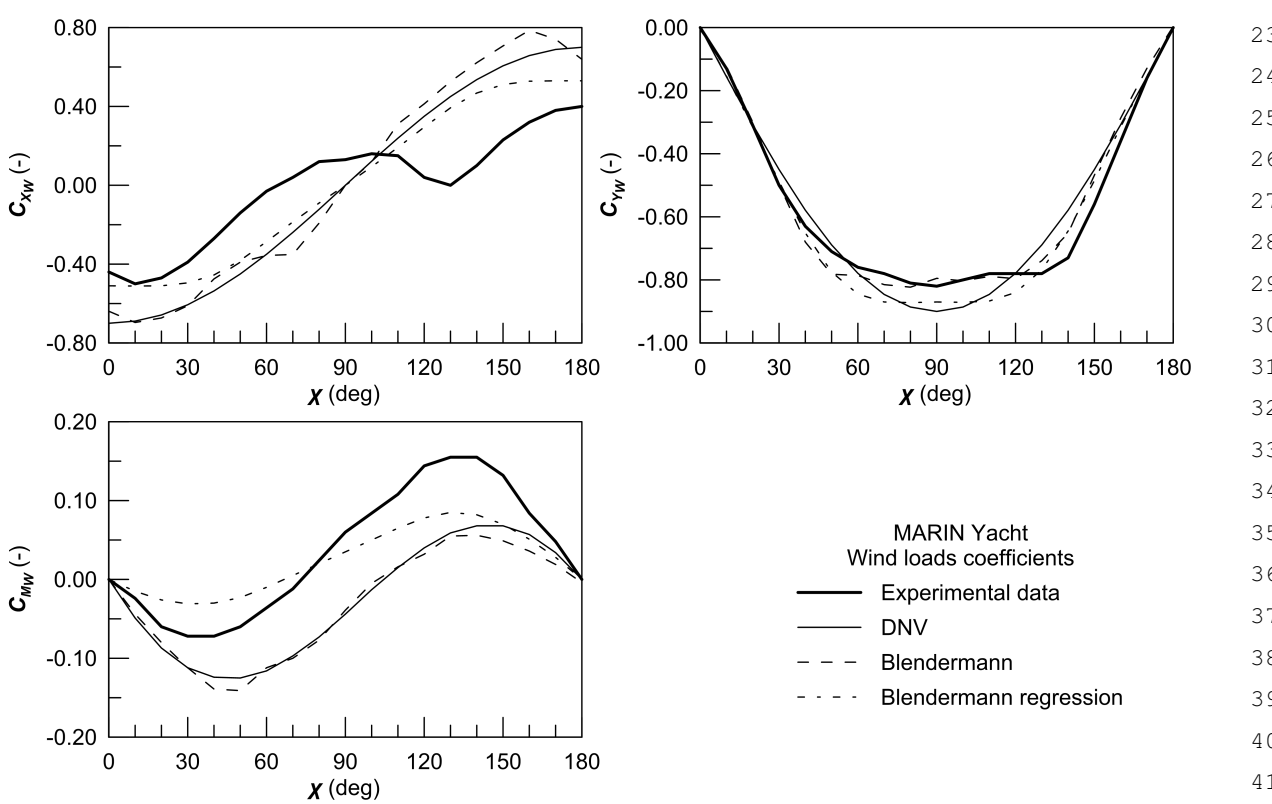

Fig. 7. Wind loads coefficients for the MARIN yacht: experimental data and empiric-statistics methods. 

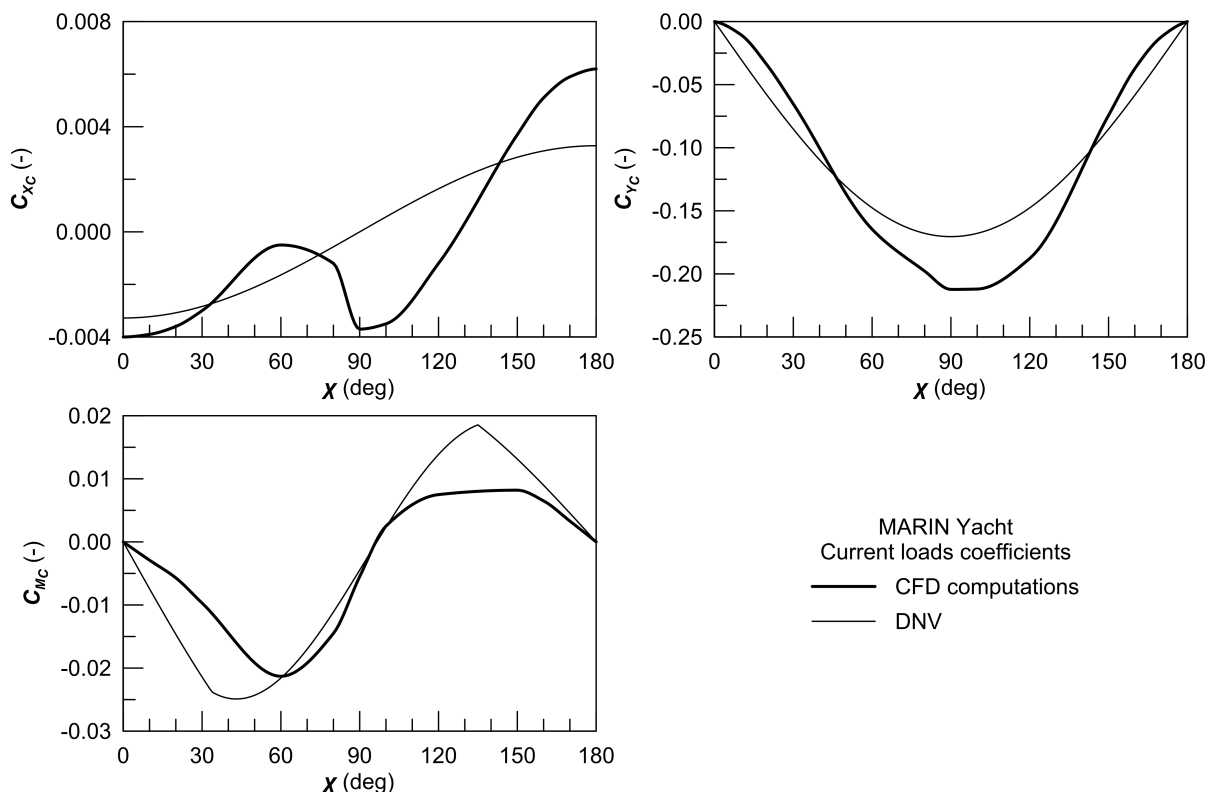

Fig. 8. Current loads coefficients for the MARIN yacht: CFD calculations and empiric-statistics method.
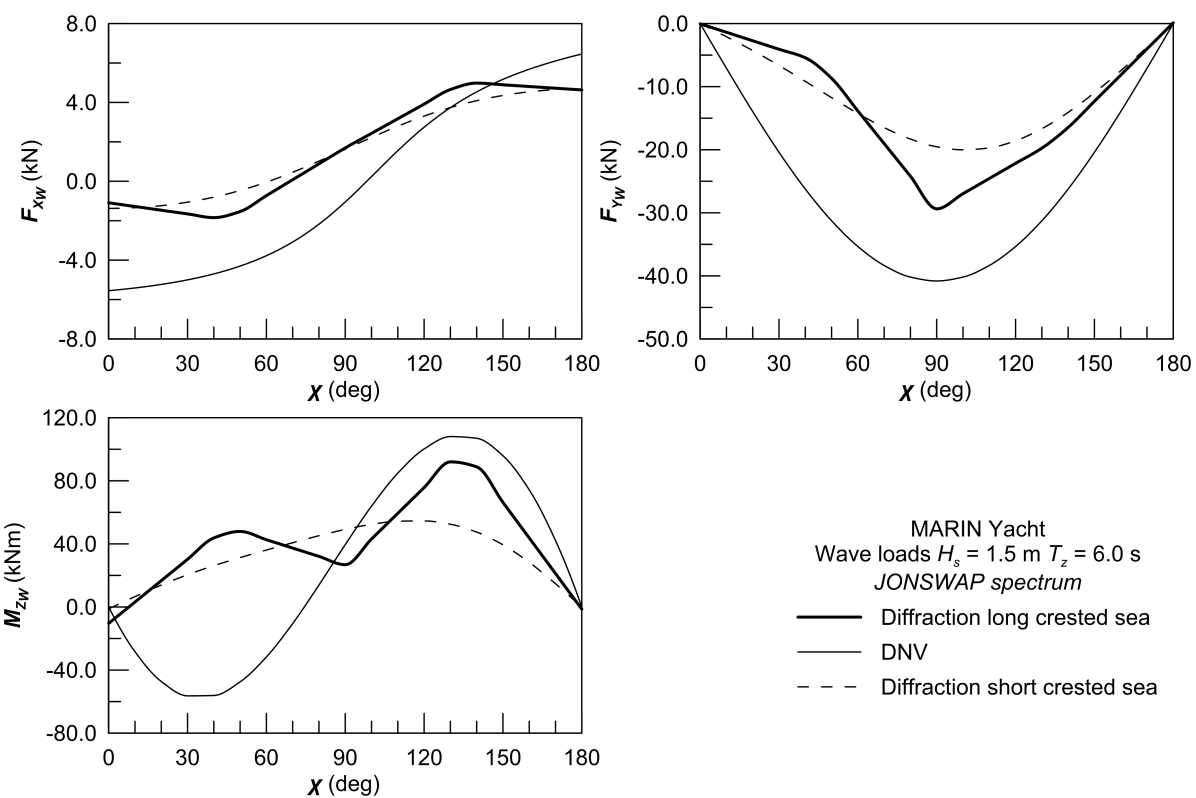

Fig. 9. Wave loads for the MARIN yacht: diffraction calculations and empiric-statistics method.

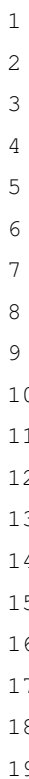



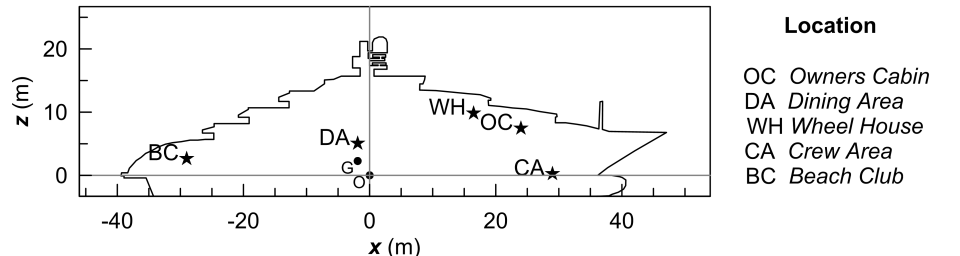

Position (from O)

$\begin{array}{rrrr}x(\mathrm{~m}) & y(\mathrm{~m}) & z(\mathrm{~m}) & 2 \\ 24.00 & 0.00 & 7.49\end{array}$

$\begin{array}{lll}-1.88 & 0.00 & 5.09\end{array}$

$\begin{array}{lll}16.30 & 0.00 & 9.89\end{array}$

$\begin{array}{llll}9.00 & 2.00 & 0.29 \\ 29.00 & 0.00 & 2.69\end{array}$

Fig. 10. Locations for comfort analysis on the MARIN yacht.
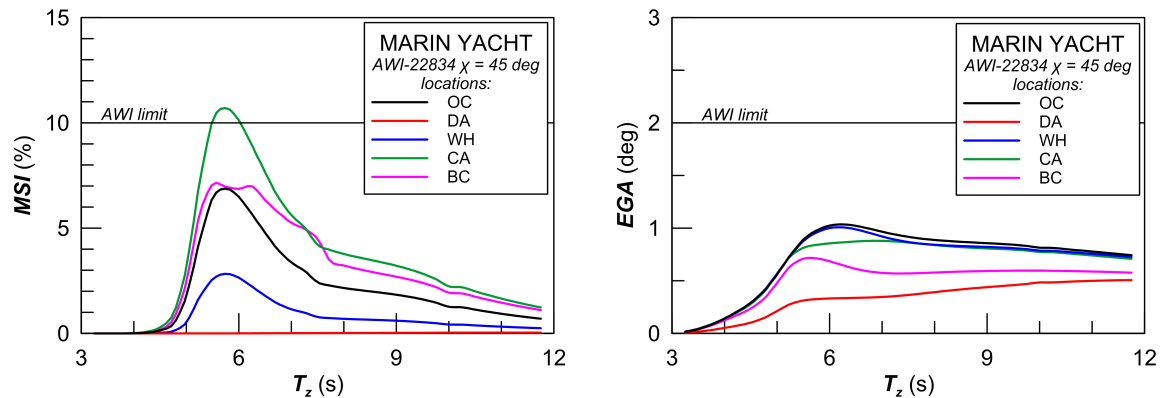

$T_{z}(\mathrm{~s})$

Fig. 11. MSI and EGA evaluation for MARIN yacht according to AWI-22834 guidelines.
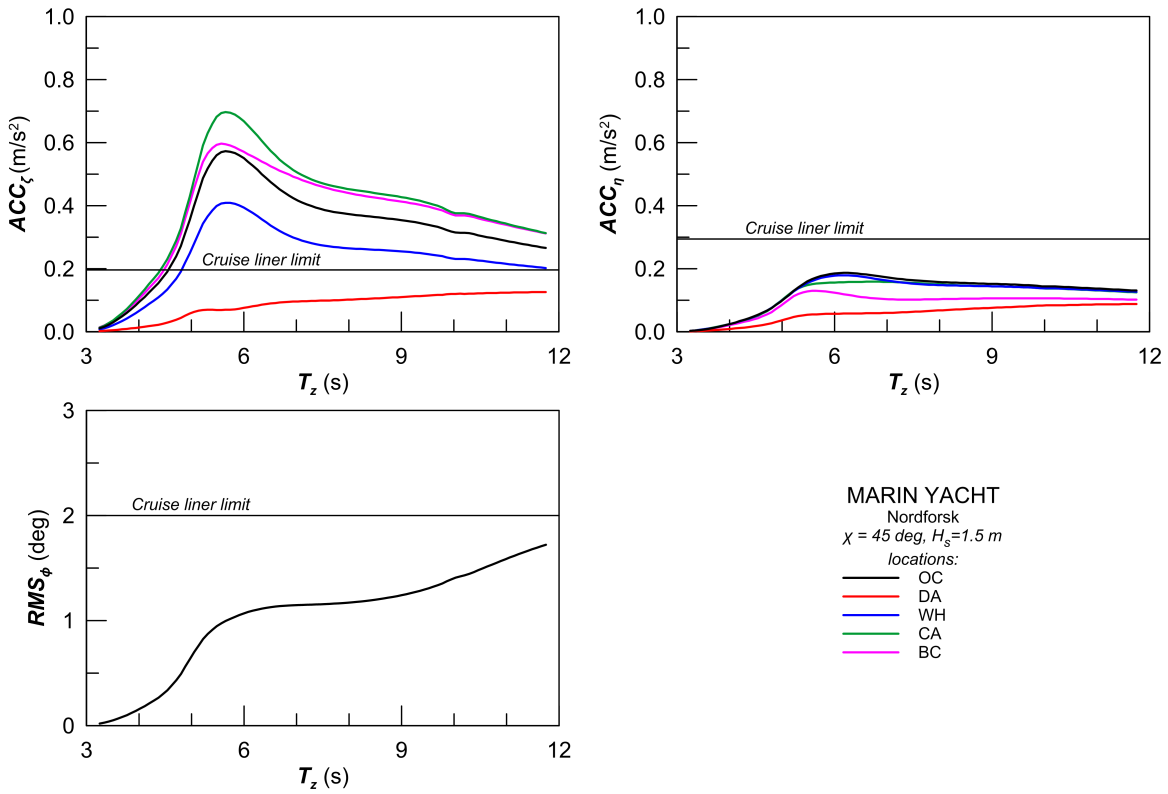

Fig. 12. Application of Nordforsk comfort criteria to the MARIN yacht for the AWI-22834 environmental conditions. 

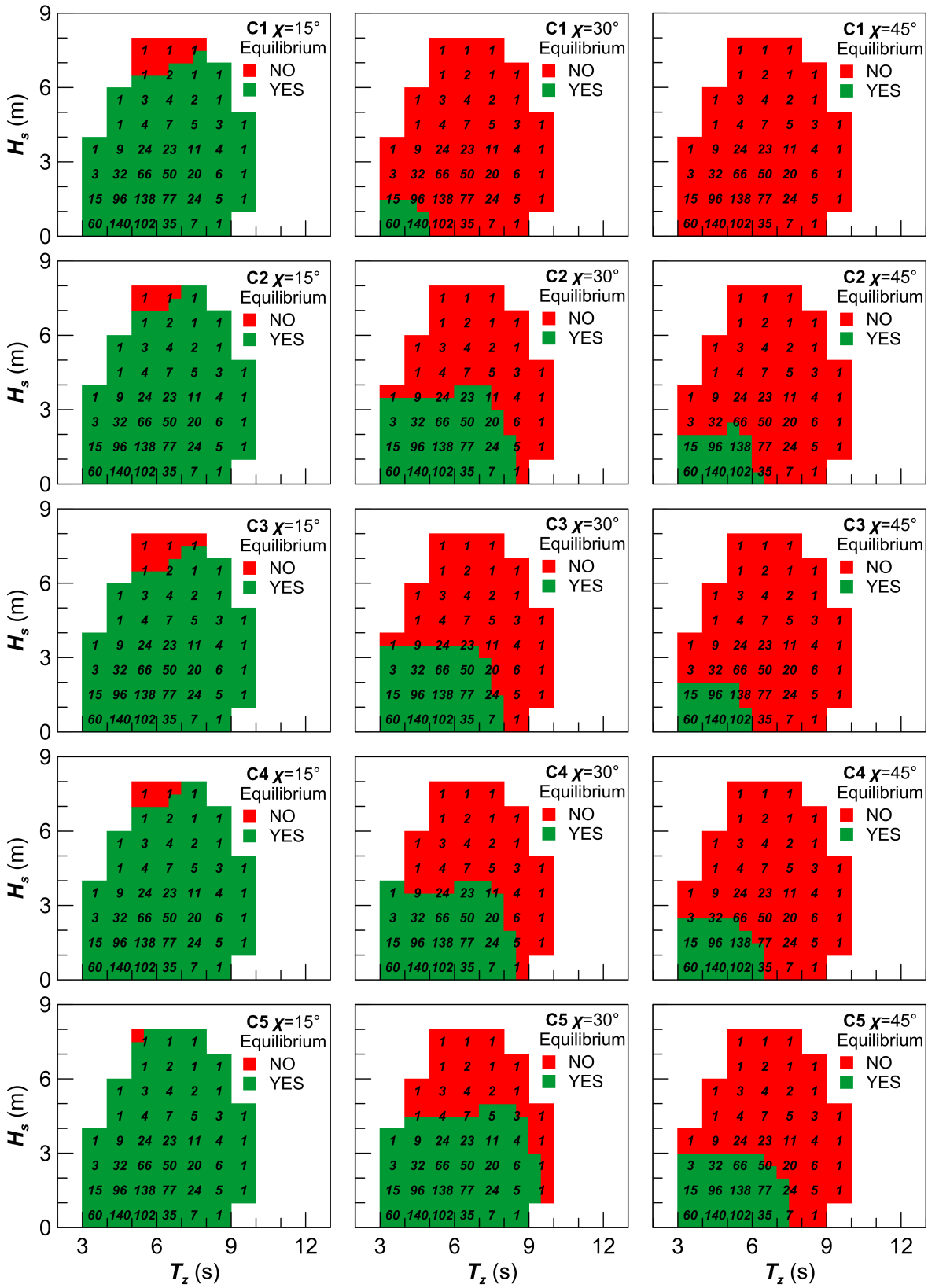

Fig. 14. Scatter diagram comparison between DP system configurations for MARIN yacht in area 26. 